\title{
A Hybrid Resynthesis Model for Hammer-String Interaction of Piano Tones
}

\author{
Julien Bensa \\ Laboratoire de Mécanique et d'Acoustique, Centre National de la Recherche Scientifique (LMA-CNRS), \\ 13402 Marseille Cedex 20, France \\ Email: bensa@lma.cnrs-mrs.fr \\ Kristoffer Jensen \\ Datalogisk Institut, Københavns Universitet, Universitetsparken 1, 2100 København, Denmark \\ Email:krist@diku.dk \\ Richard Kronland-Martinet \\ Laboratoire de Mécanique et d'Acoustique, Centre National de la Recherche Scientifique (LMA-CNRS), \\ 13402 Marseille Cedex 20, France \\ Email: kronland@lma.cnrs-mrs.fr
}

Received 7 July 2003; Revised 9 December 2003

\begin{abstract}
This paper presents a source/resonator model of hammer-string interaction that produces realistic piano sound. The source is generated using a subtractive signal model. Digital waveguides are used to simulate the propagation of waves in the resonator. This hybrid model allows resynthesis of the vibration measured on an experimental setup. In particular, the nonlinear behavior of the hammer-string interaction is taken into account in the source model and is well reproduced. The behavior of the model parameters (the resonant part and the excitation part) is studied with respect to the velocities and the notes played. This model exhibits physically and perceptually related parameters, allowing easy control of the sound produced. This research is an essential step in the design of a complete piano model.
\end{abstract}

Keywords and phrases: piano, hammer-string interaction, source-resonator model, analysis/synthesis.

\section{INTRODUCTION}

This paper is a contribution to the design of a complete piano-synthesis model. (Sound examples obtained using the method described in this paper can be found at www.lma.cnrs-mrs.fr/ kronland/JASP/sounds.html.) It is the result of several attempts $[1,2]$, eventually leading to a stable and robust methodology. We address here the modeling for synthesis of a key aspect of piano tones: the hammerstring interaction. This model will ultimately need to be linked to a soundboard model to accurately simulate piano sounds.

The design of a synthesis model is strongly linked to the specificity of the sounds to be produced and to the expected use of the model. This work was done in the framework of the analysis-synthesis of musical sounds; we seek both reconstructing a given piano sound and using the synthesis model in a musical context. The perfect reconstruction of given sounds is a strong constraint: the synthesis model must be designed so that the parameters can be extracted from the analysis of natural sounds. In addition, the playing of the synthesis model requires a good relationship between the physics of the instrument, the synthesis parameters, and the generated sounds. This relationship is crucial to having a good interaction between the "digital instrument" and the player, and it will constitute the most important aspects our piano model has to deal with.

Music based on the so-called "sound objects"-like electro-acoustic music or "musique concrète"-lies on synthesis models allowing subtle and natural transformations of the sounds. The notion of natural transformation of sounds consists here in transforming them so that they correspond to a physical modification of the instrument. As a consequence, such sound transformations calls for the model to include physical descriptions of the instrument. Nevertheless, the physics of musical instruments is sometimes too complicated to be exhaustively taken into account, or not modeled well enough to lead to satisfactory sounds. This is the case of the piano, for which hundreds of mechanical components are connected [3], and for which 
the hammer-string interaction still poses physical modeling problems.

To take into account the necessary simplifications made in the physical description of the piano sounds, we have used hybrid models that are obtained by combining physical and signal synthesis models $[4,5]$. The physical model simulates the physical behavior of the instrument whereas the signal model seeks to recreate the perceptual effect produced by the instrument. The hybrid model provides a perceptually plausible resynthesis of a sound as well as intimate manipulations in a physically and perceptually relevant way. Here, we have used a physical model to simulate the linear string vibration, and a physically informed signal model to simulate the nonlinear interaction between the string and the hammer.

An important problem linked to hybrid models is the coupling of the physical and the signal models. To use a source-resonator model, the source and the resonator must be uncoupled. Yet, this is not the case for the piano since the hammer interacts with the strings during 2 to 5 milliseconds $[6,7]$. A significant part of the piano sound characteristics is due to this interaction. Even though this observation is true from a physical point of view, this short interaction period is not in itself of great importance from a perceptual point of view. The attack is constituted of two parts due to two vibrating ways [8]: one percussive, a result of the impact of the key on the frame, and another that starts when the hammer strikes the strings. Schaeffer [9] showed that cutting the first milliseconds of a piano sound (for a bass note, for which the impact of the key on the frame is less perceptible) does not alter the perception of the sound. We have informally carried out such an experiment by listening to various piano sounds cleared of their attack. We found that, from a perceptual point of view, when the noise due to the impact of the key on the frame is not too great (compared to the vibrating energy provided by the string), the hammer-string interaction is not audible in itself. Nevertheless, this interaction undoubtedly plays an important role as an initial condition for the string motion. This is a substantial point justifying the dissociation of the string model and the source model in the design of our synthesis model. Thus, the resulting model consists in what is commonly called a "source-resonant" system (as illustrated in Figure 1). Note that the model still makes sense for high-frequency notes, for which the impact noise is of importance. Actually, the hammer-string interaction only lasts a couple of milliseconds, while the impact sound consists of an additional sound, which can be simulated using predesigned samples. Since waves are still running in the resonator after the release of the key, repeated keystroke is naturally taken into account by the model.

Laroche and Meillier [10] used such a source-resonator technique for the synthesis of piano sound. They showed that realistic piano tones can be produced using IIR filters to model the resonator and common excitation signals for several notes. Their simple resonator model, however, yielded excitation signals too long (from 4 to 5 seconds) to accurately reproduce the piano sound. Moreover, that model took into account neither the coupling between strings nor the dependence of the excitation on the velocity and octave vari-

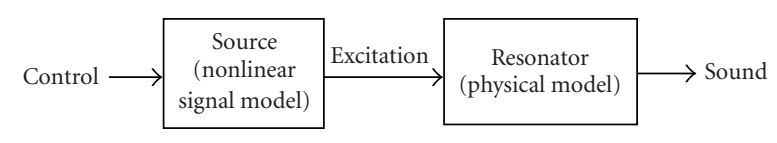

Figure 1: Hybrid model of piano sound synthesis.

ations. Smith proposed efficient resonators [11] by using the so-called digital waveguide. This approach simulates the physics of the propagating waves in the string. Moreover, the waveguide parameters are naturally correlated to the physical parameters, making for easy control. Borin and Bank $[12,13]$ used this approach to design a synthesis model of piano tones based on physical considerations by coupling digital waveguides and a "force generator" simulating the hammer impact. The commuted synthesis concept $[14,15,16]$ uses the linearity of the digital waveguide to commute and combine elements. Then, for the piano, a hybrid model was proposed, combining digital waveguide, a phenomenological hammer model, and a time-varying filtering that simulates the soundboard behavior. Our model is an extension of these previous works, to which we added a strong constraint of resynthesis capability. Here, the resonator was modeled using a physically related model, the digital waveguide; and the source-destined to generate the initial condition for the string motion-was modeled using a signal-based nonlinear model.

The advantages of such a hybrid model are numerous:

(i) it is simple enough so that the parameters can be accurately estimated from the analysis of real sound,

(ii) it takes into account the most relevant physical characteristics of the piano strings (including coupling between strings) and it permits the playing to be controlled (the velocity of the hammer),

(iii) it simulates the perceptual effect due to the nonlinear behavior of the hammer-string interaction, and it allows sounds transformation with both physical and perceptual approaches.

Even though the model we propose is not computationally costly, we address here its design and its calibration rather than its real time implementation. Hence, the calculus and reasoning are done in the frequency domain. The time domain implementation should give rise to a companion article.

\section{THE RESONATOR MODEL}

Several physical models of transverse wave propagation on a struck string have been published in the literature $[17,18,19$, $20]$. The string is generally modeled using a one-dimensional wave equation. The specific features of the piano string that are important in wave propagation (dispersion due to the stiffness of the string and frequency-dependent losses) are further incorporated through several perturbation terms. To account for the hammer-string interaction, this equation is then coupled to a nonlinear force term, leading to a system of equations for which an analytical solution cannot be 
exhibited. Since the string vibration is transmitted only to the radiating soundboard at the bridge level, it is not useful to numerically calculate the entire spatial motion of the string. The digital waveguide technique [11] provides an efficient way of simulating the vibration at the bridge level of the string, when struck at a given location by the hammer. Moreover, the parameters of such a model can be estimated from the analysis of real sounds [21].

\subsection{The physics of vibrating strings}

We present here the main features of the physical modeling of piano strings. Consider the propagation of transverse waves in a stiff damped string governed by the motion equation [21]

$$
\frac{\partial^{2} y}{\partial t^{2}}-c^{2} \frac{\partial^{2} y}{\partial x^{2}}+\kappa^{2} \frac{\partial^{4} y}{\partial x^{4}}+2 b_{1} \frac{\partial y}{\partial t}-2 b_{2} \frac{\partial^{3} y}{\partial x^{2} \partial t}=P(x, t)
$$

where $y$ is the transverse displacement, $c$ the wave speed, $\kappa$ the stiffness coefficient, $b_{1}$ and $b_{2}$ the loss parameters. Frequency-dependent loss is introduced via mixed timespace derivative terms (see $[21,22]$ for more details). We apply fixed boundary conditions

$$
\left.y\right|_{x=0}=\left.y\right|_{x=L}=\left.\frac{\partial^{2} y}{\partial x^{2}}\right|_{x=0}=\left.\frac{\partial^{2} y}{\partial x^{2}}\right|_{x=L}=0,
$$

where $L$ is the length of the string. After the hammer-string contact, the force $P$ is equal to zero and this system can be solved. An analytical solution can be expressed as a sum of exponentially damped sinusoids:

$$
y(x, t)=\sum_{n=1}^{\infty} a_{n}(x) e^{-\alpha_{n} t} e^{i \omega_{n} t}
$$

where $a_{n}$ is the amplitude, $\alpha_{n}$ is the damping coefficient, and $\omega_{n}$ is the frequency of the $n$th partial. Due to the stiffness, the waves are dispersed and the partial frequencies, which are not perfectly harmonic, are given by [23]

$$
\omega_{n}=2 \pi n \omega_{0} \sqrt{1+B n^{2}}
$$

where $\omega_{0}$ is the fundamental radial frequency of the string without stiffness, and $B$ is the inharmonicity coefficient [23]. The losses are frequency dependent and expressed by [21]

$$
\alpha_{n}=-b_{1}-b_{2}\left(\frac{\pi^{2}}{2 B L^{2}}\left[-1+\sqrt{1+4 B\left(\frac{\omega_{n}}{\omega_{0}}\right)^{2}}\right]\right)
$$

The spectral content of the piano sound, and of most musical instruments, is modified with respect to the dynamics. For the piano, this nonlinear behavior consists of an increase of the brightness of the sound and it is linked mainly to the hammer-string contact (the nonlinear nature of the generation of longitudinal waves also participates in the increase of brightness; we do not take this phenomena into account since we are interested only in transversal waves). The stiff-

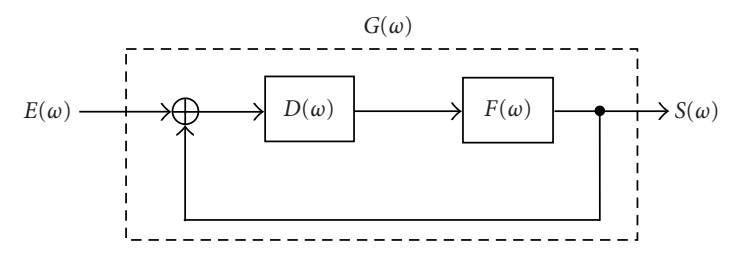

FIgURE 2: Elementary digital waveguide (named $G$ ).

ness of the hammer felt increases with the impact velocity. In the next paragraph, we show how the waveguide model parameters are related to the amplitudes, damping coefficients, and frequencies of each partial.

\subsection{Digital waveguide modeling}

\subsubsection{The single string case: elementary digital waveguide}

To model wave propagation in a piano string, we use a digital waveguide model [11]. In the single string case, the elementary digital waveguide model (named $G$ ) we used consists of a single loop system (Figure 2) including

(i) a delay line (a pure delay filter named $D$ ) simulating the time the waves take to travel back and forth in the medium,

(ii) a filter (named $F$ ) taking into account the dissipation and dispersion phenomena, together with the boundary conditions. The modulus of $F$ is then related to the damping of the partials and the phase to inharmonicity in the string,

(iii) an input $E$ corresponding to the frequency-dependent energy transferred to the string by the hammer,

(iv) an output $S$ representing the vibrating signal measured at an extremity of the string (at the bridge level).

The output of the digital waveguide driven by a delta function can be expanded as a sum of exponentially damped sinusoids. The output thus coincides with the solution of the motion equation of transverse waves in a stiff damped string for a source term given by a delta function force. As shown in $[21,24]$, the modulus and phase of $F$ are related to the damping and the frequencies of the partials by the expressions

$$
\begin{gathered}
\left|F\left(\omega_{n}\right)\right|=e^{\alpha_{n} D}, \\
\arg \left(F\left(\omega_{n}\right)\right)=\omega_{n} D-2 n \pi,
\end{gathered}
$$

with $\omega_{n}$ and $\alpha_{n}$ given by (4) and (5).

After some calculations (see [21]), we obtain the expressions of the modulus and the phase of the loop filter in terms of the physical parameters:

$$
\begin{gathered}
|F(\omega)| \simeq \exp \left(-D\left[b_{1}+\frac{b_{2} \pi^{2} \xi}{2 B L^{2}}\right]\right), \\
\arg (F(\omega)) \simeq D \omega-D \omega_{0} \sqrt{\frac{\xi}{2 B}},
\end{gathered}
$$


with

$$
\xi=-1+\sqrt{1+\frac{4 B \omega^{2}}{\omega_{0}^{2}}}
$$

in terms of the inharmonicity coefficient $B$ [23].

\subsubsection{The multiple strings case: coupled digital waveguides}

In the middle and the treble range of the piano, there are two or three strings for each note in order to increase the efficiency of the energy transmission towards the bridge. The vibration produced by this coupled system is not the superposition of the vibrations produced by each string. It is the result of a complex coupling between the modes of vibration of these strings [25]. This coupling leads to phenomena like beats and double decays on the amplitude of the partials, which constitute one of the most important features of the piano sound. Beats are used by professionals to precisely tune the doublets or triplets of strings. To resynthesize the vibration of several strings at the bridge level, we use coupled digital waveguides. Smith [14] proposed a coupling model with two elementary waveguides. He assumed that the two strings were coupled to the same termination, and that the losses were lumped to the bridge impedance. This technique leads to a simple model necessitating only one loss filter. But the decay times and the coupling of the modes are not independent. Välimäki et al. [26] proposed another approach that couples two digital waveguides through real gain amplifiers. In that case, the coupling is the same for each partial, and the time behavior of the partials is similar. For synthesis purpose, Bank [27] showed that perceptually plausible beating sound can be obtained by adding only a few resonators in parallel.

We have designed two models, a two- and a threecoupled digital waveguides, which are an extension of Välimäki et al.'s approach. They consist in separating the time behavior of the components by using complex-valued and frequency-dependent linear filters to couple the waveguides. The three-coupled digital waveguide is shown on Figure 3. The two models accurately simulate the energy transfer between the strings (see Section 2.4.3). A related method [28] (with an example of piano coupling) has been recently available in the context of digital waveguide networks.

Each string is modeled using an elementary digital waveguide (named $G_{1}, G_{2}, G_{3}$; each loop filter and delays are named $F_{1}, F_{2}, F_{3}$, and $D_{1}, D_{2}, D_{3}$ respectively). The coupled model is then obtained by connecting the output of each elementary waveguide to the input of the others through coupling filters. The coupling filters simulate the wave propagation along the bridge and are thus correlated to the distance between the strings. In the case of a doublet of strings, the two coupling filters (named $C$ ) are identical. In the case of a triplet of strings, the coupling filters of adjacent strings (named $C_{a}$ ) are equal but differ from the coupling filters of the extreme strings (named $C_{e}$ ). The excitation signal is assumed to be the same for each elementary waveguide since we suppose the hammer strikes the strings in a similar way.
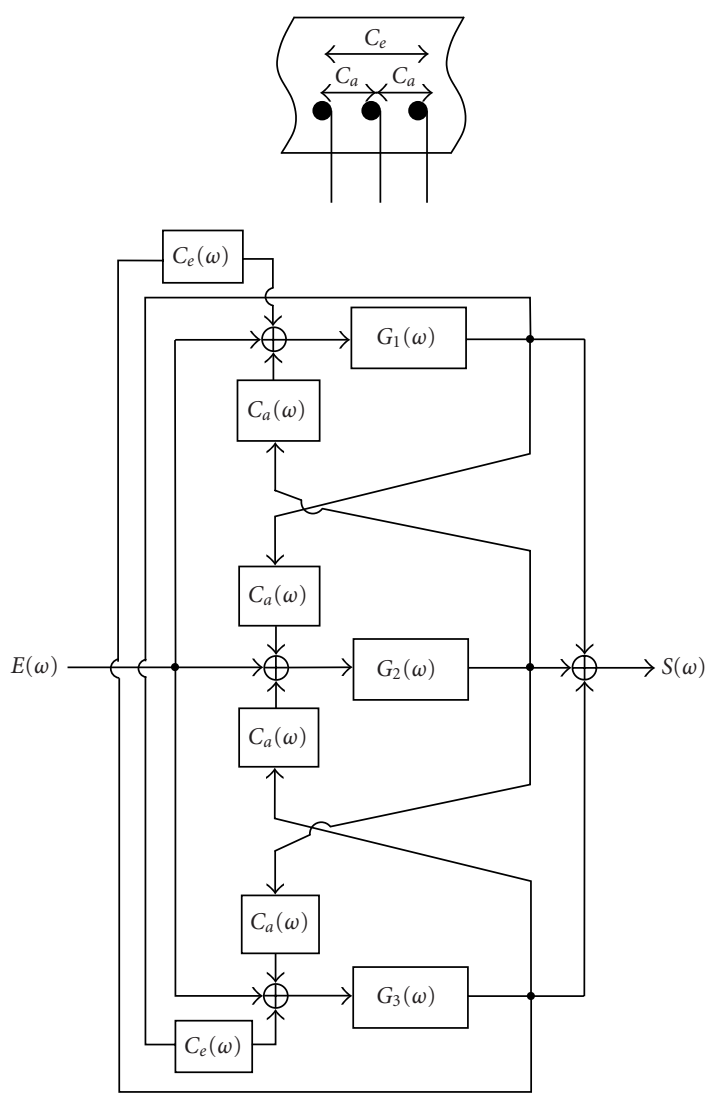

Figure 3: The three-coupled digital waveguide (bottom) and the corresponding physical system at the bridge level (top).

To ensure the stability of the different models, one has to respect specific relations. First the modulus of the loop filters must be inferior to 1 . Second, for coupled digital waveguides, the following relations must be verified:

$$
|C| \sqrt{\left|G_{1}\right|\left|G_{2}\right|}<1
$$

in the case of two-coupled waveguides, and

$$
\left|G_{1} G_{2} C_{a}^{2}+G_{1} G_{3} C_{e}^{2}+G_{2} G_{3} C_{a}^{2}+2 G_{1} G_{2} G_{3} C_{a}^{2} C_{e}\right|<1
$$

in the case of three-coupled waveguides. Assuming that those relations are verified, the models are stable.

This work takes place in the general analysis-synthesis framework, meaning that the objective is not only to simulate sounds, but also to reconstruct a given sound. The model must therefore be calibrated carefully. In the next section is presented the inverse problem allowing the waveguide parameters to be calculated from experimental data. We then describe the experiment and the measurements for one-, two- and three-coupled strings. We then show the validity and the accuracy of the analysis-synthesis process by comparing synthetic and original signals. Finally, the behavior of the signal of the real piano is verified. 


\subsection{The inverse problem}

We address here the estimation of the parameters of each elementary waveguide as well as the coupling filters from the analysis of a single signal (measured at the bridge level). For this, we assume that in the case of three-coupled strings the signal is composed of a sum of three exponentially decaying sinusoids for each partial (and respectively one and two exponentially decaying sinusoids in the case of one and two strings). The estimation method is a generalization of the one described in [29] for one and two strings. It can be summarized as follows: start by isolating each triplet of the measured signal through bandpass filtering (a truncated Gaussian window); then use the Hilbert transform to get the corresponding analytic signal and obtain the average frequency of the component by derivating the phase of this analytic signal; finally, extract from each triplet the three amplitudes, damping coefficients, and frequencies of each partial by a parametric method (Steiglitz-McBride method [30]).

The second part of the process is described in detail in the appendix. In brief, we identify the Fourier transform of the sum of the three exponentially damped sinusoids (the measured signal) with the transfer function of the digital waveguide (the model output). This identification leads to a linear system that admits an analytical solution in the case of one or two strings. In the case of three coupled strings, the solution can be found only numerically. The process gives an estimation of the modulus and of the phase of each filter near the resonance peaks as a function of the amplitudes, damping coefficients, and frequencies. Once the resonator model is known, we extract the excitation signal by a deconvolution process with respect to the waveguide transfer function. Since the transfer function has been identified near the resonant peaks, the excitation is also estimated at discrete frequency values corresponding to the partial frequencies. This excitation corresponds to the signal that has to be injected into the resonator to resynthesize the actual sound.

\subsection{Analysis of experimental data and validation of the resonator model}

We describe here first an experimental setup allowing the measurement of the vibration of one, two, or three strings struck by a hammer for different velocities. Then we show how to estimate the resonator parameters from those measurements, and finally, we compare original and synthesized signals. This experimental setup is an essential step that validates the estimation method. Actually, estimating the parameters of one-, two-, or three-coupled digital waveguides from only one signal is not a trivial process. Moreover, in a real piano, many physical phenomena are not taken into account in the model presented in the previous section. It is then necessary to verify the validity of the model on a laboratory experiment before applying the method to the piano case.

\subsubsection{Experimental setup}

On the top of a massive concrete support, we have attached a piece of a bridge taken from a real piano. On the other extremity of the structure, we have attached an agraffe on

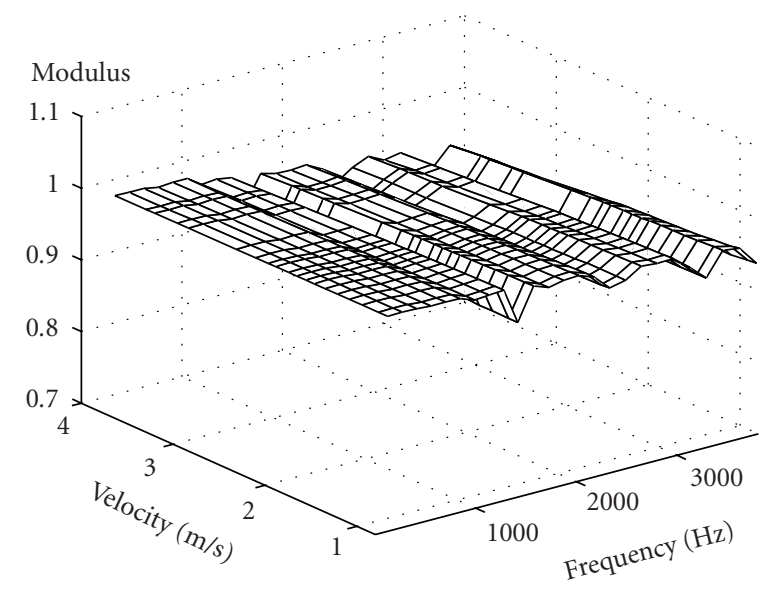

Figure 4: Amplitude of filter $F$ as a function of the frequency and of hammer velocity.

a hardwood support. The strings are tightened between the bridge and the agraffe and tuned manually. It is clear that the strings are not totally uncoupled to their support. Nevertheless, this experiment has been used to record signals of struck strings, in order to validate the synthesis models, and was it entirely satisfactory for this purpose. One, two, or three strings are struck with a hammer linked to an electronically piloted key. By imposing different voltages to the system, one can control the hammer velocity in a reproducible way. The precise velocity is measured immediately after escapement by using an optic sensor (MTI 2000, probe module $2125 \mathrm{H}$ ) pointing to the side of the head of the hammer. The vibration at the bridge level is measured by an accelerometer (B\&K 4374). The signals are directly recorded on digital audio tape. Acceleration signals correspond to hammer velocities between $0.8 \mathrm{~m} . \mathrm{s}^{-1}$ and $5.7 \mathrm{~m} . \mathrm{s}^{-1}$.

\subsubsection{Filter estimation}

From the signals collected on the experimental setup, a set of data was extracted. For each hammer velocity, the waveguide filters and the corresponding excitation signals were estimated using the techniques described above. The filters were studied in the frequency domain; it is not the purpose of this paper to describe the method for the time domain and to fit the transfer function using IIR or FIR filters.

Figure 4 shows the modulus of the filter response $F$ for the first twenty-five partials in the case of tones produced by a single string. Here the hammer velocity varies from $0.7 \mathrm{~m} . \mathrm{s}^{-1}$ to $4 \mathrm{~m} . \mathrm{s}^{-1}$. One notices that the modulus of the waveguide filters is similar for all hammer velocities. The resonator represents the strings that do not change during the experiment. If the estimated resonator remains the same for different hammer velocities, all the nonlinear behavior due to the dynamic has been taken into account in the excitation part. The resonator and the source are well separated. This result validates our approach based on a source-resonator separation. For high frequency partials, however, the filter modulus decreased slightly as a function of the hammer velocity. This nonlinear behavior is not directly linked to the 


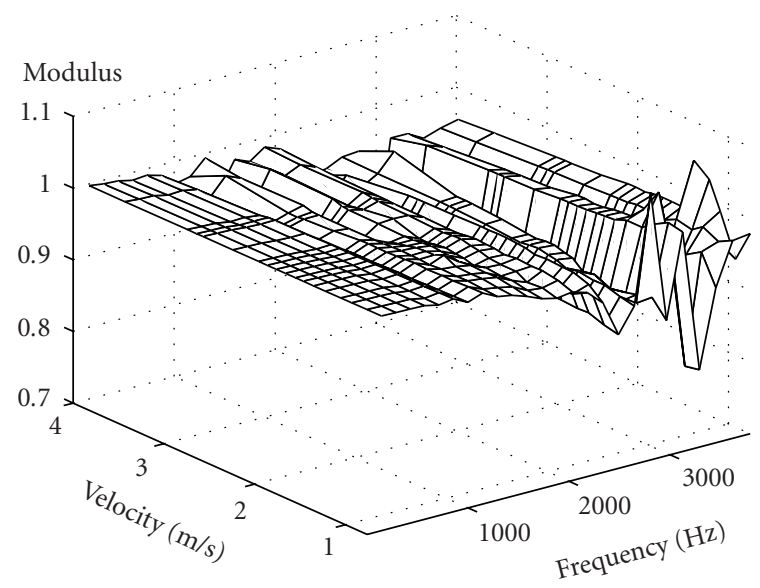

FIgURE 5: Amplitude of filter $F_{2}$ (three-coupled waveguide model) as a function of the frequency and of hammer velocity.

hammer-string contact. It is mainly due to nonlinear phenomena involved in the wave propagation. At large amplitude motion, the tension modulation introduces greater internal losses (this effect is even more pronounced in plucked strings than in struck strings).

The filter modulus slowly decreases (as a function of frequency) from a value close to 1 . Since the higher partials are more damped than the lower ones, the amplitude of the filter decreases as the frequency increases. The value of the filter modulus (close to 1) suggests that the losses are weak. This is true for the piano string and is even more obvious on this experimental setup, since the lack of a soundboard limits the acoustic field radiation. More losses are expected in the real piano.

We now consider the multiple strings case. From a physical point of view, the behavior of the filters $F_{1}, F_{2}$, and $F_{3}$ (which characterize the intrinsic losses) of the coupled digital waveguides should be similar to the behavior of the filter $F$ for a single string, since the strings are supposed identical. This is verified except for high-frequency partials. This behavior is shown on Figure 5 for filter $F_{2}$ of the three-coupled waveguide model. Some artifacts pollute the drawing at high frequencies. The poor signal/noise ratio at high frequency (above $2000 \mathrm{~Hz}$ ) and low velocity introduce error terms in the analysis process, leading to mistakes on the amplitudes of the loop filters (for instance, a very small value of the modulus of one loop filter may be compensated by a value greater than one for another loop filter; the stability of the coupled waveguide is then preserved). Nevertheless, this does not alter the synthetic sound since the corresponding partials (high frequency) are weak and of short duration.

The phase is also of great importance since it is related to the group delay of the signal and consequently directly linked to the frequency of the partials. The phase is a nonlinear function of the frequency (see (8)). It is constant with the hammer velocity (see Figure 6) since the frequencies of the partials are always the same (linearity of the wave propagation).

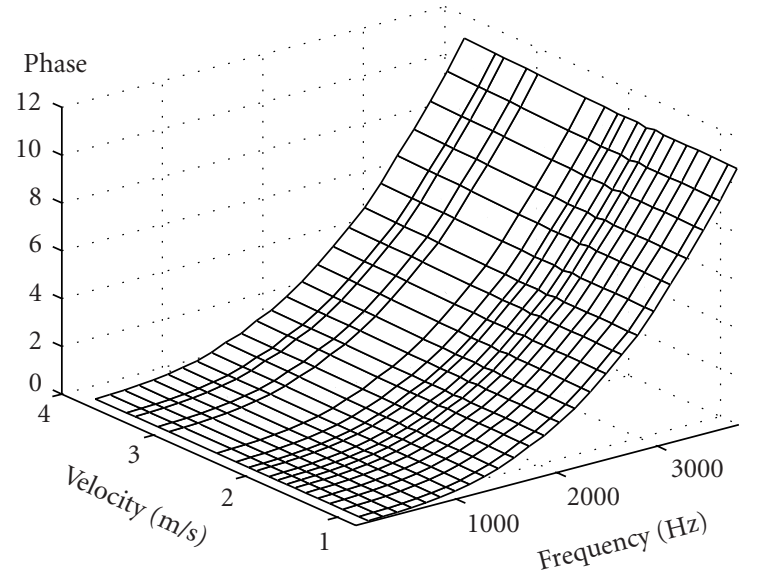

Figure 6: Phase of filter $F$ as a function of the frequency and hammer velocity.

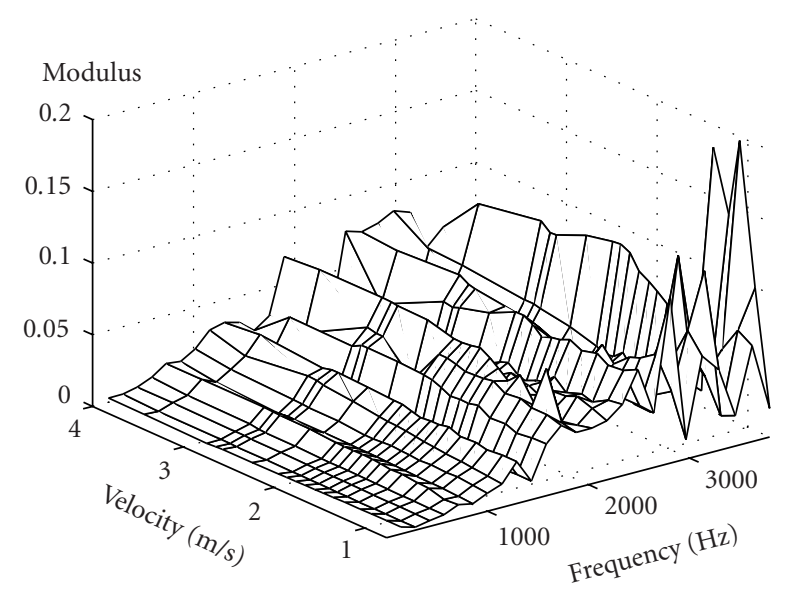

FIgUre 7: Modulus of filter $C_{a}$ as a function of the frequency and of hammer velocity.

The coupling filters simulate the energy transfer between the strings and are frequency dependent. Figure 7 represents one of these coupling filters for different values of the hammer velocity. The amplitude is constant with respect to the hammer velocity (up to signal/noise ratio at high frequency and low velocity), showing that the coupling is independent of the amplitude of the vibration. The coupling rises with the frequency. The peaks at frequencies $700 \mathrm{~Hz}$ and $1300 \mathrm{~Hz}$ correspond to a maximum.

\subsubsection{Accuracy of the resynthesis}

At this point, one can resynthesize a given sound by using a single- or multicoupled digital waveguide and the parameters extracted from the analysis. For the synthetic sounds to be identical to the original requires describing the filters precisely. The model was implemented in the frequency domain, as described in Section 2, thus taking into account the exact amplitude and the phase of the filters (for instance, for a three-coupled digital waveguide, we have to implement three 


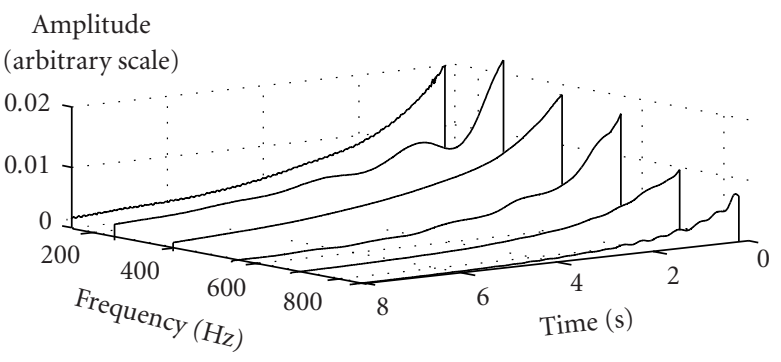

(a)

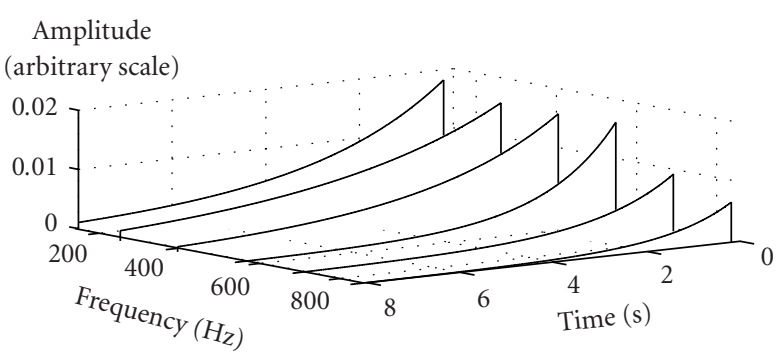

(b)

FIgURE 8: Amplitude modulation laws (velocity of the bridge) for the first six partials, one string, of the (a) original and (b) resynthesised sound.

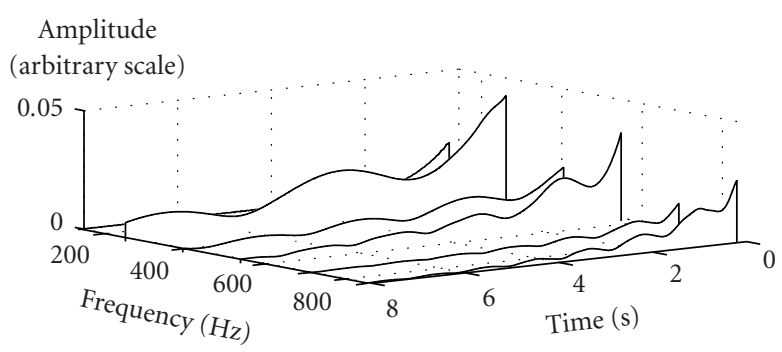

(a)

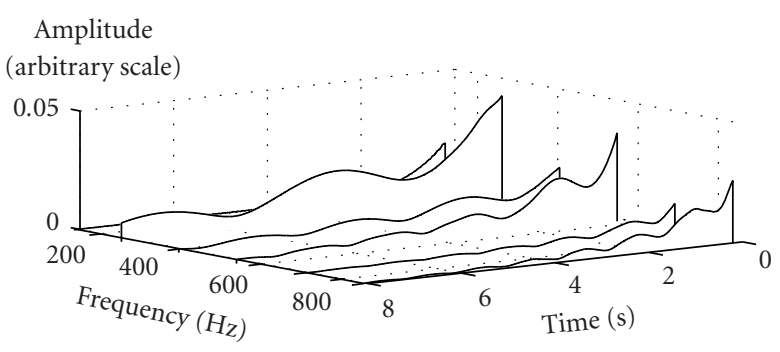

(b)

FIgURe 9: Amplitude modulation laws (velocity of the bridge) for the first six partials, two strings, of the (a) original and (b) resynthesised sound.

delays and five complex filters, moduli, and phases). Nevertheless, for real-time synthesis purposes, filters can be approached by IIR of low order (see, e.g., [26]). This aspect will

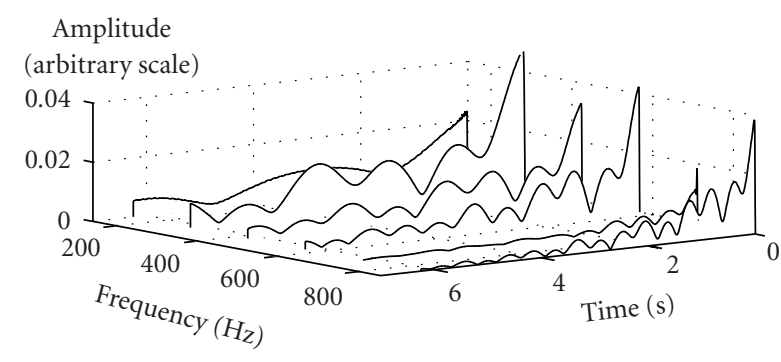

(a)

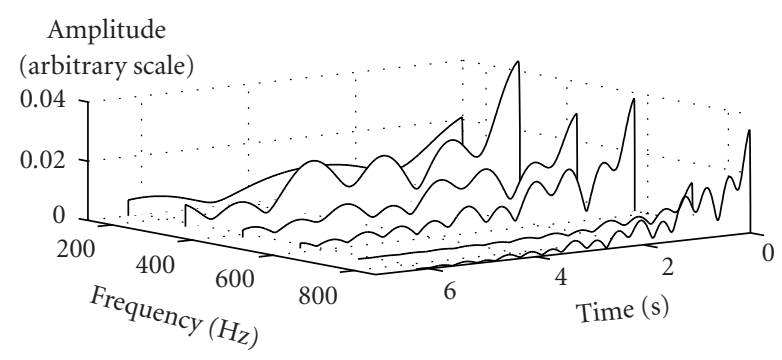

(b)

FIgure 10: Amplitude modulation laws (velocity of the bridge) for the first six partials, three strings, of the (a) original and (b) resynthesised sound.

be developed in future reports. By injecting the excitation signal obtained by deconvolution into the waveguide model, the signal measured is reproduced on the experimental setup. Figures 8,9 , and 10 show the amplitude modulation laws (velocity of the bridge) of the first six partials of the original and the resynthesized sound. The variations of the temporal envelope are generally well retained, and for the coupled system (in Figures 9 and 10), the beat phenomena are well reproduced. The slight differences, not audible, are due to fine physical phenomena (coupling between the horizontal and the vertical modes of the string) that are not taken into account in our model.

In the one-string case, we now consider the second and sixth partials of the original sound in Figure 8. We can see beats (periodic amplitude modulations) that show coupling phenomena on only one string. Indeed, the horizontal and vertical modes of vibration of the string are coupled through the bridge. This coupling was not taken into account in this study since the phenomenon is of less importance than coupling between two different strings. Nevertheless, we have shown in [29] that coupling between two modes of vibration can also be simulated using a two-coupled digital waveguide model. The accuracy of the resynthesis validates a posteriori our model and the source-resonator approach.

\subsection{Behavior and control of the resonator through measurements on a real piano}

To take into account the note dependence of the resonator, we made a set of measurements on a real piano, a Yamaha Disklavier C6 grand piano equipped with sensors. The 


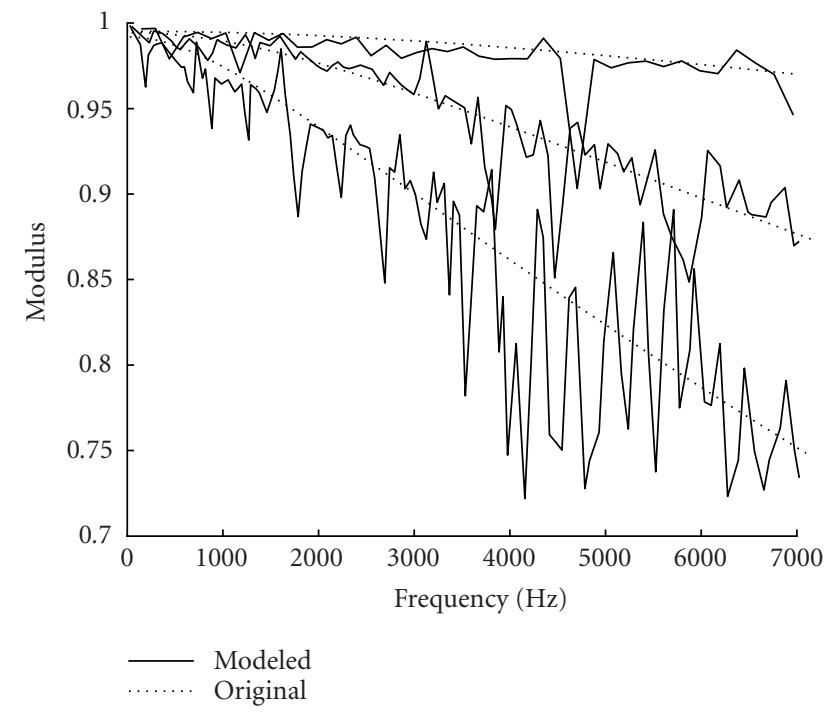

FIgURE 11: Modulus of the waveguide filters for notes A0, F1 and D3, original and modeled.

vibrations of the strings were measured at the bridge by an accelerometer, and the hammer velocities were measured by a photonic sensor. Data were collected for several velocities and several notes. We used the estimation process described in Section 2.3 for the previous experimental setup and extracted for each note and each velocity the corresponding resonator and source parameters.

As expected, the behavior of the resonator as a function of the hammer velocity and for a given note is similar to the one described in Section 2.4.2, for the signals measured on the experimental setup. The filters are similar with respect to the hammer velocity. Their modulus is close to one, but slightly weaker than previously, since it now takes into account the losses due to the acoustic field radiated by the soundboard. The resynthesis of the piano measurements through the resonator model and the excitation obtained by deconvolution are perceptively satisfactory since the sound is almost indistinguishable from the original one.

On the contrary, the shape of the filters is modified as a function of the note. Figure 11 shows the modulus of the waveguide filter $F$ for several notes (in the multiple string case, we calculated an average filter by arithmetic averaging). The modulus of the loop filter is related to the losses undergone by the wave over one period. Note that this modulus increases with the fundamental frequency, indicating decreasing loss over one period as the treble range is approached.

The relations (7) and (8), relating the physical parameters to the waveguide parameters, allow the resonator to be controlled in a relevant physical way. We can either change the length of the strings, the inharmonicity, or the losses. But to be in accordance with the physical system, we have to take into account the interdependence of some parameters. For instance, the fundamental frequency is obviously related to the length of the string, and to the tension and the linear mass. If we modify the length of the string, we also have to

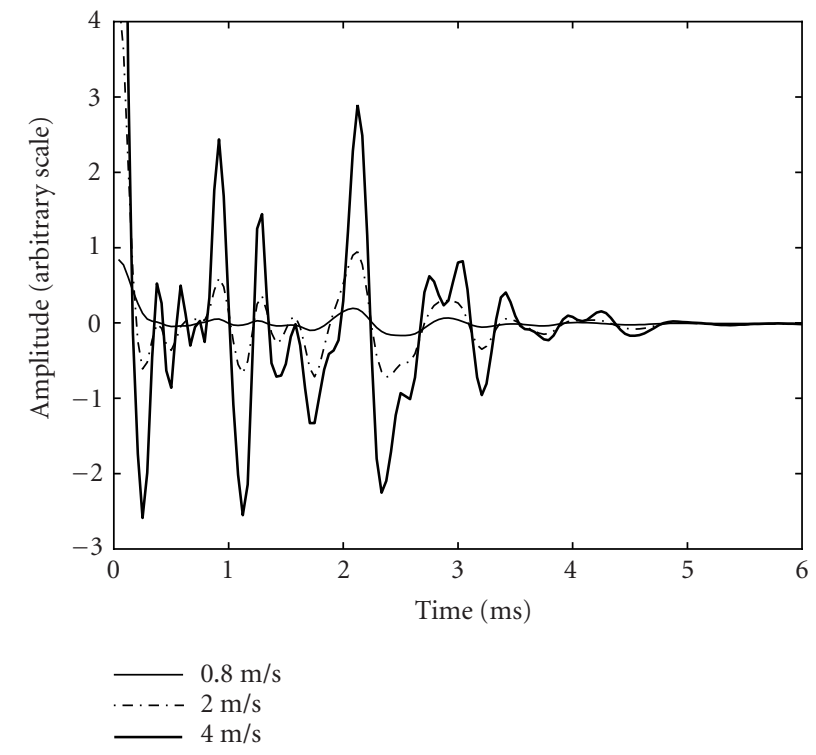

FIGURE 12: Waveform of three excitation signals of the experimental setup, corresponding to three different hammer velocities.

modify, for instance, the fundamental frequency, considering that the tension and the linear mass are unchanged. This aspect has been taken into account in the implementation of the model.

\section{THE SOURCE MODEL}

In the previous section, we observed that the waveguide filters are almost invariant with respect to the velocity. In contrast, the excitation signals (obtained as explained in Section 2.3 and related to the impact of the hammer on the string) varies nonlinearly as a function of the velocity, thereby taking into account the timbre variations of the resulting piano sound. From the extracted excitation signals, we here study the behavior and design a source model by using signal methods, so as to simulate these behaviors precisely. The source signal is then convolved with the resonator filter to obtain the piano bridge signal.

\subsection{Nonlinear source behavior as a function of the hammer velocity}

Figure 12 shows the excitation signals extracted from the measurement of the vibration of a single string struck by a hammer for three velocities corresponding to the pianissimo, mezzo-forte, and fortissimo musical playing. The excitation duration is about 5 milliseconds, which is shorter than what Laroche and Meillier [10] proposed and in accordance with the duration of the hammer-string contact [6]. Since this interaction is nonlinear, the source also behaves nonlinearly. Figure 13 shows the spectra of several excitation signals obtained for a single string at different velocities regularly spaced between 0.8 and $4 \mathrm{~m} / \mathrm{s}$. The excitation corresponding to fortissimo provides more energy than the ones corresponding to mezzo-forte and pianissimo. But this increased 


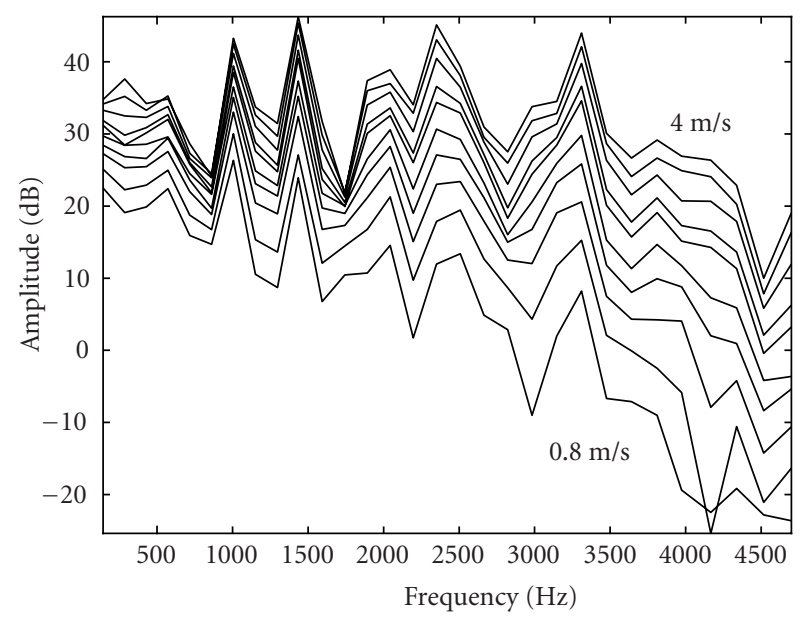

FIgURE 13: Amplitude of the excitation signals for one string and several velocities.

amplitude is frequency dependent: the higher partials increase more rapidly than the lower ones with the same hammer velocity. This increase in the high partials corresponds to an increase in brightness with respect to the hammer velocity. It can be better visualized by considering the spectral centroid [31] of the excitation signals. Figure 14 shows the behavior of this perceptually (brightness) relevant criteria [32] as a function of the hammer velocity. Clearly, for one, two, or three strings, the spectral centroid is increased, corresponding to an increased brightness of the sound. In addition to the change of slope, which translates into the change of brightness, Figure 13 shows several irregularities common to all velocities, among which a periodic modulation related to the location of the hammer impact on the string.

\subsection{Design of a source signal model}

The amplitude of the excitation increases smoothly as a function of the hammer velocity. For high-frequency components, this increase is greater than for low frequency components, leading to a flattening of the spectrum. Nevertheless, the general shape of the spectrum stays the same. Formants do not move and the modulation of the spectrum due to the hammer position on the string is visible at any velocity. These observations suggest that the behavior of the excitation could be well reproduced using a subtractive synthesis model.

The excitation signal is seen as an invariant spectrum shaped by a smooth frequency response filter, the characteristics of which depend on the hammer velocity. The resulting source model is shown on Figure 15. The subtractive source model consists of the static spectrum, the spectral deviation, and the gain. The static spectrum takes into account all the information that is invariant with respect to the hammer velocity. It is a function of the characteristics of the hammer and the strings. The spectral deviation and the gain both shape the spectrum as function of the hammer velocity. The spectral deviation simulates the shifting of the energy to the high frequencies, and the gain models the global increase of

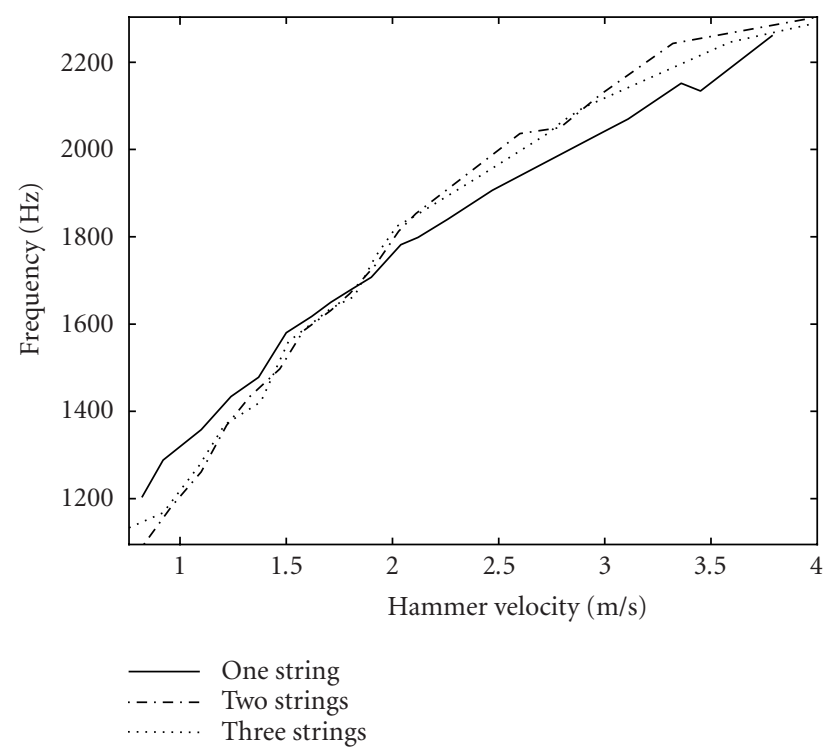

Figure 14: The spectral centroid of the excitation signals for one (plain), two (dash-dotted) and three (dotted) strings.

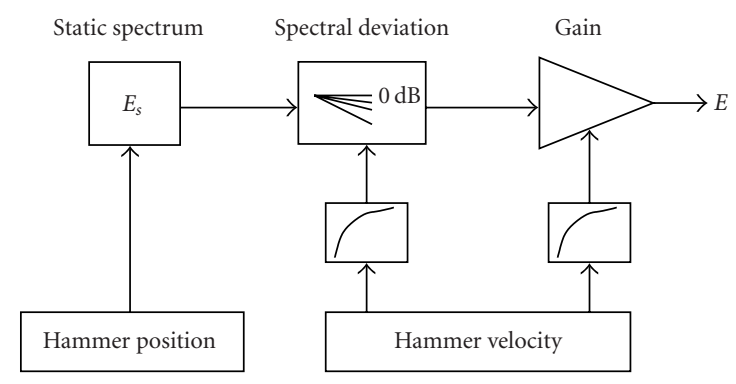

FIGURE 15: Diagram of the subtractive source model.

amplitude. Earlier versions of this model were presented in $[1,2]$. This type of models has been, in addition, shown to work well for many instruments [33].

In the early days of digital waveguides, Jaffe and Smith [24] modeled the velocity-dependent spectral deviation as a one-pole lowpass filter. Laursen et al. [34] proposed a second-order biquad filter to model the differences between guitar tones with different dynamics.

A similar approach was developed by Smith and Van Duyne in the time domain [15]. The hammer-string interaction force pulses were simulated using three impulses passed through three lowpass filters which depend on the hammer velocity. In our case, a more accurate method is needed to resynthesize the original excitation signal faithfully.

\subsubsection{The static spectrum}

We defined the static spectrum as the part of the excitation that is invariant with the hammer velocity. Considering the expression of the amplitude of the partials, $a_{n}$, for a hammer striking a string fixed at its extremities (see Valette and Cuesta [19]), and knowing that the spectrum of the excitation is 


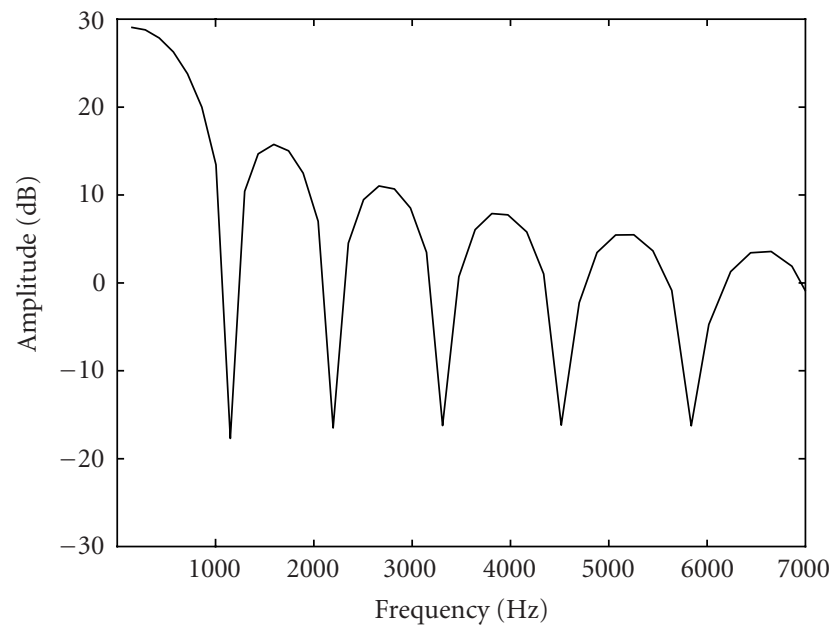

Figure 16: The static spectrum $E_{s}(\omega)$.

related to amplitudes of the partials by $E=a_{n} D$ [29], the static spectrum $E_{s}$ can be expressed as

$$
E_{s}\left(\omega_{n}\right)=\frac{4 L}{T} \frac{\sin \left(n \pi x_{0} / L\right)}{n \pi \sqrt{1+n^{2} B}}
$$

where $T$ is the string tension and $L$ its length, $B$ is the inharmonicity factor, and $x_{0}$ the striking position. We can easily measure the striking position, the string length and the inharmonicity factor on our experimental setup. On the other hand, we have an only estimation of the tension, it can be calculated through the fundamental frequency and the linear mass of the string.

Figure 16 shows this static spectrum for a single string. Many irregularities, however, are not taken into account for several reasons. We will see later their importance from a perceptual point of view. Equation (12) is still used, however, when the hammer position is changed. This is useful when one plays with a different temperament because it reduces dissonance.

\subsubsection{The deviation with the dynamic}

The spectral deviation and the gain take into account the dependency of the excitation signal on velocity. They are estimated by dividing the spectrum of the excitation signal by the static spectrum for all velocities:

$$
d(\omega)=\frac{E(\omega)}{E_{s}(\omega)},
$$

where $E$ is the original excitation signal. Figure 17 shows this deviation for three hammer velocities. It effectively strengthens the fortissimo, in particular for the medium and high partials. Its evolution with the frequency is regular and can successfully be fitted to a first-order exponential polynomial (as shown in Figure 17)

$$
\hat{d}=\exp (a f+g),
$$

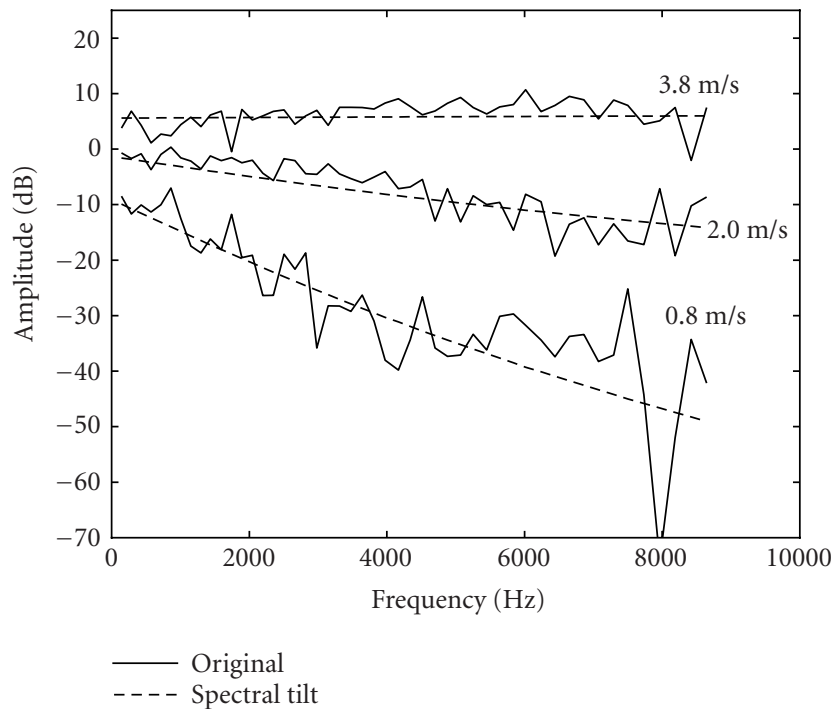

FIGURE 17: Dynamic deviation of three excitation signals of the experimental setup, original and modeled.
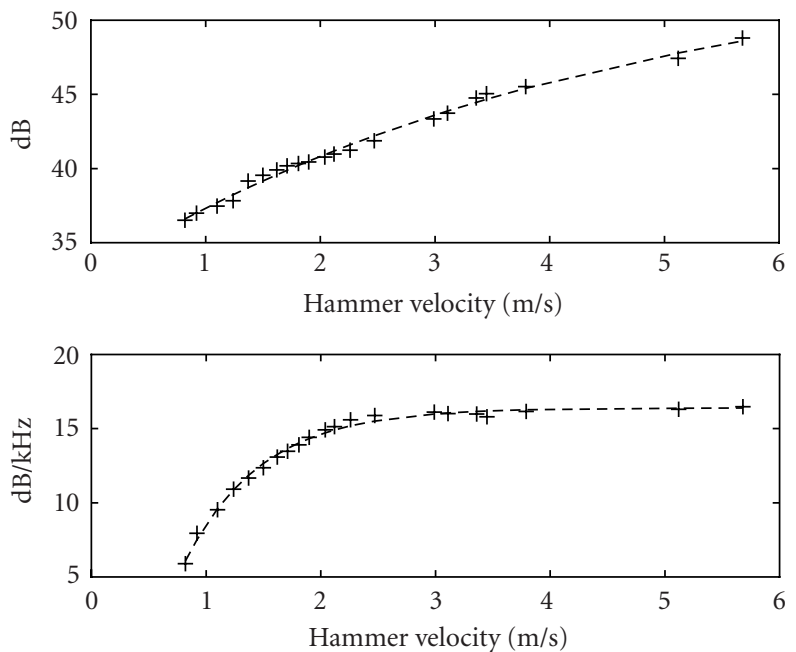

Figure 18: Parameters $g$ (gain)(top), $a$ (spectral deviation) (bottom) as a function of the hammer velocity for the experimental setup signals, original $(+)$ and modeled (dashed).

where $\hat{d}$ is the modeled deviation. The term $g$ corresponds to the gain (independent of the frequency) and the term $a f$ corresponds to the spectral deviation. The variables $g$ and $a$ depend on the hammer velocity. To get a usable source model, we must consider the parameter's behavior with different dynamics. Figure 18 shows the two parameters for several hammer velocities. The model is consistent since their behavior is regular. But the tilt increases with the hammer velocity, showing an asymptotic and nonlinear behavior. This observation can be directly related to the physics of the hammer. As we have seen, when the felt is compressed, it becomes harder and thus gives more energy to high frequencies. But, for high velocities, the felt is totally compressed and its hardness is almost constant. Thus, the amplitude of the 
corresponding string wave increases further but its spectral content is roughly the same. We have fitted this asymptotic behavior by an exponential model (see Figure 18), for each parameter $g$ and $a$,

$$
\begin{aligned}
& g(v)=\alpha_{g}-\beta_{g} \exp \left(-\gamma_{g} v\right), \\
& a(v)=\alpha_{a}-\beta_{a} \exp \left(-\gamma_{a} v\right),
\end{aligned}
$$

where $\alpha_{i}(i=g, a)$ is the asymptotic value, $\beta_{i}(i=g, a)$ is the deviation from the asymptotic value at zero velocity (the dynamic range), and $\gamma_{i}(i=g, a)$ is the velocity exponential coefficient, governing how sensitive the attribute is to a velocity change. The parameters of this exponential model were found using a nonlinear weighted curvefit.

\subsubsection{Resynthesis of the excitation signal}

For a given velocity, the excitation signal can now be recreated using (13), (14), and (15). The inverse Fourier transform of this source model convoluted with the transfer function of the resonator leads to a realistic sound of a string struck by a hammer. The increase in brightness with the dynamic is well reproduced. But from a resynthesis point of view, this model is not satisfactory. The reproduced signal is different from the original one; it sounds too regular and monotonous. To understand this drawback of our model, we calculated the error we made by dividing the original excitation signal by the modeled one for each velocity. The corresponding curves are shown on Figure 19 for three velocities.

Notice that this error term does not depend on the hammer velocity, meaning that our static spectrum model is too straightforward and does not take into account the irregularities of the original spectrum. Irregularities are due to many phenomena including the width of the hammer-string contact, hysteretic phenomena in the felt, nonlinear phenomena in the string, and mode resonances of the hammer. To obtain a more realistic sound with our source model, we include this error term in the static spectrum. The resulting original and resynthesized signals are shown on Figure 20. The deviations of the resulting excitations are perceptually insignificant. The synthesized sound obtained is then close to the original one.

\subsection{Behavior and control of the source through measurements on a real piano}

The source model parameters were calculated for a subset of the data for the piano, namely the notes A0, F1, B1, G2, C3, G3, D4, E5, and F6. Each note has approximately ten velocities, from about $0.4 \mathrm{~m} / \mathrm{s}$ to between 3 to $6 \mathrm{~m} / \mathrm{s}$. The source extracted from the signals measured on the piano behaves as the data obtained with the experimental setting for all notes with respect to the hammer velocity. The dynamic deviation is well modeled by the gain $g$ and the spectral deviation parameter $a$. As in Section 3.2, their behavior as a function of the velocity is well fitted using an asymptotic exponential curve.

From a perceptual point of view, an increased hammer velocity corresponds both to an increased loudness and a rel-

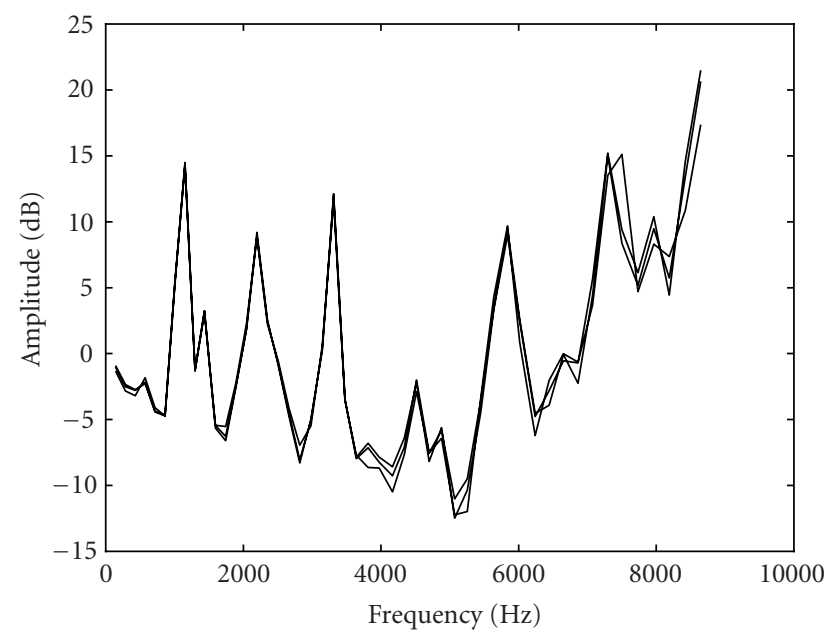

FIGURE 19: Example of the error spectrum. The large errors generally fall in the weak parts of the spectrum.

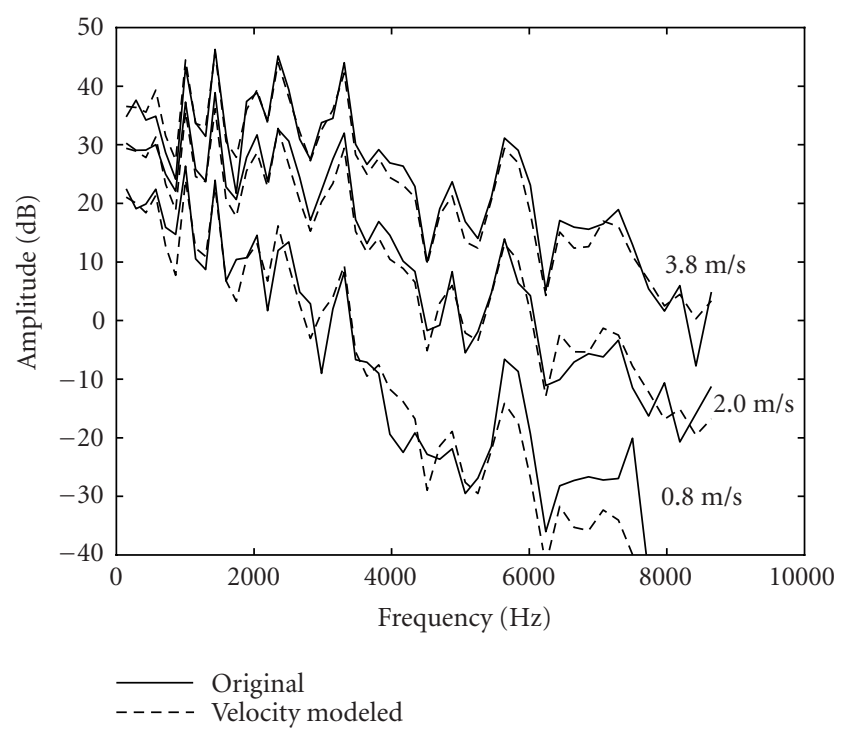

FIGURE 20: Original and modeled excitation spectrum for three different hammer velocities for the experimental setup signals.

ative increase in high frequencies leading to a brighter tone. Equations (15) make it possible to resynthesize of the excitation signal for a given note and hammer velocity. However, parameters $g$ and $a$ used in the modeling are linked in a complex way to the two most important perceptual features of the tone, that is, loudness and brightness. Thus, without a thorough knowledge of the model, the user will not be able to adjust the parameters of the virtual piano to obtain a satisfactory tone. To get an intuitive control of the model, the user needs to be provided access to these perceptual parameters, loudness and brightness, closely corresponding to energy and spectral centroid. The energy $E n$ is directly correlated to the perception of loudness and the spectral centroid $B a$ to the 
perception of brightness [32]. These parameters are given by

$$
\begin{aligned}
& E n=\frac{1}{T} \int_{0}^{F s / 2} E^{2}(f) d f, \\
& B a=\frac{\int_{0}^{F s / 2} E(f) f d f}{\int_{0}^{F s / 2} E(f) d f},
\end{aligned}
$$

where $f$ is the frequency and $F s$ the sampling frequency.

To synthesize an excitation signal having a given energy and spectral centroid, we must express parameters $g$ and $a$ as functions of $B a$ and $E n$. The centroid actually depends only on $a$ :

$$
B a=\frac{\int_{0}^{F s / 2} E s(f) e^{a f} f d f}{\int_{0}^{F s / 2} E s(f) e^{a f} d f} .
$$

We numerically calculate the expression of $a$ as a function of $B a$ and store the solution in a table. Alternatively, assuming that the brightness change is unaffected by the shape of the static spectrum $E s$, the spectral deviation parameter $a$ can be calculated directly from the given brightness [35].

Knowing $a$, we can calculate $g$ from the energy $E n$ by the relation

$$
g=\frac{1}{2} \log \left(\frac{E n T}{\int_{0}^{F s / 2} E s^{2}(f) e^{2 a f^{2}+2 b f}}\right) .
$$

The behavior of $B a$ and $E n$ as a function of the hammer velocity will then determine the dynamic range of the instrument and it must be defined by the user.

Figure 21 shows the behavior of the spectral centroid and the energy for several notes. The curves have similar behavior and differ mainly by a multiplicative constant. We have fitted their asymptotic behavior by an exponential model, similarly to what was done with (15). These functions are applied to the synthesis of each excitation signal and then characterize the dynamic range of the virtual instrument. It is easy for the user to change the dynamic range of the virtual instrument, which is modified by the user by changing the shape of these functions.

Calculating the excitation signal is then done as follows. To a given note and velocity, we associate a spectral centroid $B a$ and an energy $E n$ (using the asymptotic exponential fit); $a$ is then obtained from the spectral centroid and $g$ from the energy (equation (18)). One finally gets the spectral deviation which, multiplied by the static spectrum, allows the excitation signal to be calculated.

\section{CONCLUSION}

The reproduction of the piano bridge vibration is undoubtly the first most important step for piano sound synthesis. We show that a hybrid model consisting of a resonant part and an excitation part is well adapted for this purpose. After accurate calibration, the sounds obtained are perceptually close to the original ones for all notes and velocities. The resonator, which simulates the phenomena intervening in the strings

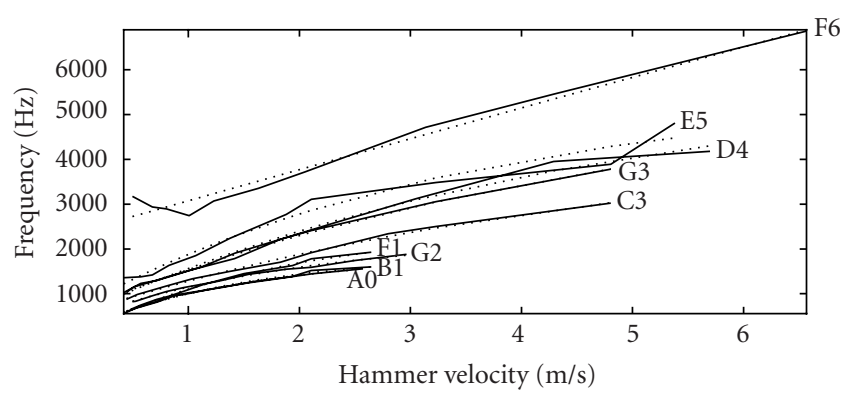

(a)

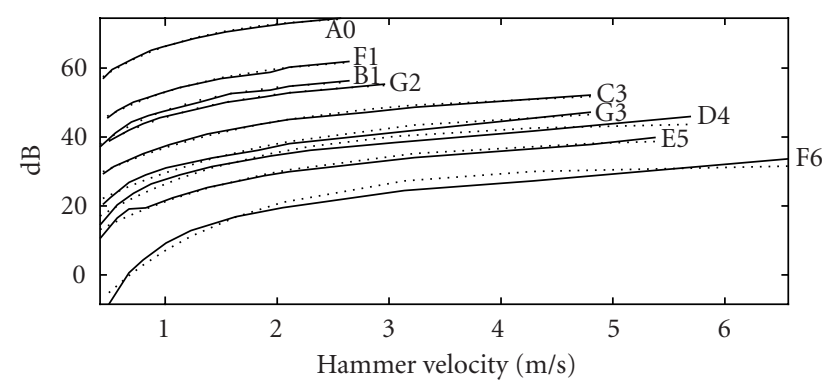

(b)

FIGURE 21: Spectral centroid (a) and energy (b) for several notes as a function of the hammer velocity, original (plain) and modeled (dotted).

themselves, is modeled by a digital waveguide model that is very efficient in simulating the wave propagation. The resonator model exhibits physical parameters such as the string tension, the inharmonicity coefficient, allowing physically relevant control of the resonator. It also takes into account the coupling effects, which are extremely relevant for perception. The source is extracted using a deconvolution process and is modeled using a subtractive signal model. The source model consists of three parts (static spectrum, spectral deviation, and gain) that are dependent on the velocities and the notes played. To get intuitive control of the source model, we exhibited two parameters: the spectral centroid and the energy, strongly related to the perceptual parameters brightness and loudness. This perceptual link permits easy control of the dynamic characteristics of the piano.

Thus, the tone of a given piano can be synthesized using a hybrid model. This model is currently implemented in realtime using a Max-MSP software environment.

\section{APPENDIX}

\section{INVERSE PROBLEM, THREE-COUPLED DIGITAL WAVEGUIDE}

We show in this appendix how the parameters of a threecoupled digital waveguide model can be expressed as function of the modal parameters. This method is an extension of the model presented in [29]. 
The signal measured at the bridge level is the result of the vibration of three coupled strings. Each partial is actually constituted by at least three components, having frequencies which are slightly different from the frequencies of each individual string. We write the measured signal as a sum of exponentially damped sinusoids:

$$
s(t)=\sum_{k=1}^{\infty} a_{1 k} e^{-\alpha_{1 k} t} e^{i \omega_{1 k} t}+a_{2 k} e^{-\alpha_{2 k} t} e^{i \omega_{2 k} t}+a_{3 k} e^{-\alpha_{3 k} t} e^{i \omega_{3 k} t},
$$

with $a_{1 k}, a_{2 k}$, and $a_{3 k}$ the initial amplitudes, $\alpha_{1 k}, \alpha_{2 k}, \alpha_{3 k}$ and $\omega_{1 k}, \omega_{2 k}, \omega_{3 k}$ the damping coefficients and the frequencies of the components of the $k$ th partial. The Fourier transform of $s(t)$ is

$$
\begin{aligned}
S(\omega)= & \sum_{k=1}^{\infty} \frac{a_{1 k}}{\alpha_{1 k}+i\left(\omega-\omega_{1} k\right)}+\frac{a_{2 k}}{\alpha_{2 k}+i\left(\omega-\omega_{2} k\right)} \\
& +\frac{a_{3 k}}{\alpha_{3 k}+i\left(\omega-\omega_{3} k\right)} .
\end{aligned}
$$

We identify this expression locally in frequency with the output $T(\omega)$ of the three-coupled waveguide model (see Figure 3):

$$
T(\omega)=\frac{N_{1}}{N_{2}}
$$

with

$$
\begin{aligned}
N_{1}= & F_{1}+F_{2}+F_{3} \\
& +2\left[\left(C_{a}-1\right)\left(F_{1} F_{2}+F_{2} F_{3}\right)+\left(C_{e}-1\right) F_{1} F_{3}\right] \\
& +F_{1} F_{2} F_{3}\left[3+4 C_{e} C_{a}-4 C_{a}-2 C_{e}-C_{e}^{2}\right], \\
N_{2}= & 1-\left(F_{1}+F_{2}+F_{3}\right)+\left(F_{1} F_{2}+F_{2} F_{3}\right)\left(1-C_{a}^{2}\right) \\
& +F_{1} F_{3}\left(1-C_{e}^{2}\right)+F_{1} F_{2} F_{3}\left(2 C_{a}^{2}+C_{e}^{2}-2 C_{a}^{2}\right. \\
& \left.+C_{e}^{2}-2 C_{a}^{2} C_{e}-1\right),
\end{aligned}
$$

where $F_{i}(i=1,2,3)$ are the loop filters of the digital waveguides $G_{i}(i=1,2,3)$ (without loss of generality, one can assume that $D_{1}=D_{2}=D_{3}=D$, since the difference in delays can be taken into account in the phase of the filter $F_{i}$ ). For this purpose, since $T(\omega)$ is a rational fraction of third-order polynomial in $e^{-i \omega D}$ (see (6)), it can be decomposed into a sum of three rational fractions of the first-order polynomial in $e^{-i \omega D}$ :

$$
\begin{aligned}
T(\omega)= & \frac{P(\omega) e^{-i \omega D}}{1-X(\omega) e^{-i \omega D}}+\frac{Q(\omega) e^{-i \omega D}}{1-Y(\omega) e^{-i \omega D}} \\
& +\frac{R(\omega) e^{-i \omega D}}{1-Z(\omega) e^{-i \omega D}} .
\end{aligned}
$$

The vibrations generated by the model are assimilated to a superposition of three series of partials whose frequencies and decay times are governed by the quantities $X(\omega), Y(\omega)$, and $Z(\omega)$. By identification between (A.3) and (A.5), we de- termine the following system of 6 equations:

$$
\begin{gathered}
P+Q+R=F_{1}+F_{2}+F_{3}, \\
P Y+P Z+Q X+Q Z+R X+R Y \\
=2 F_{1} F_{2}\left(1-C_{a}\right)+2 F_{1} F_{3}\left(1-C_{e}\right) \\
+2 F_{2} F_{3}\left(1-C_{a}\right), \\
P Y Z+Q X Z+R X Y \\
=F_{1} F_{2} F_{3}\left(4 C_{a} C_{e}-4 C_{a}-2 C_{e}-C_{e}^{2}+3\right), \\
X+Y+Z=F_{1}+F_{2}+F_{3}, \\
X Y+X Z+Y Z=F_{1} F_{2}\left(1-C_{a}^{2}\right)+F_{2} F_{3}\left(1-C_{a}^{2}\right) \\
+F_{1} F_{3}\left(1-C_{e}^{2}\right), \\
X Y Z=F_{1} F_{2} F_{3}\left(1-2 C_{a}^{2}-C_{e}^{2}+2 C_{a}^{2} C_{e}\right) .
\end{gathered}
$$

We identify (A.2) with the excitation signal times the transfer function $T$ (equation (A.5)):

$$
S(\omega)=E(\omega) T(\omega) .
$$

Assuming that two successive modes do not overlap (these assumptions are verified for the piano sound) and by writing

$$
\begin{aligned}
& X(\omega)=|X(\omega)| e^{i \Phi_{X}(\omega)}, \\
& Y(\omega)=|Y(\omega)| e^{i \Phi_{Y}(\omega),} \\
& Z(\omega)=|Z(\omega)| e^{i \Phi_{Z}(\omega)},
\end{aligned}
$$

we express (A.12) near each double resonance as

$$
\begin{aligned}
& \frac{a_{1 k}}{\alpha_{1 k}+i\left(\omega-\omega_{1} k\right)}+\frac{a_{2 k}}{\alpha_{2 k}+i\left(\omega-\omega_{2} k\right)}+\frac{a_{3 k}}{\alpha_{3 k}+i\left(\omega-\omega_{3} k\right)} \\
& \simeq \frac{E(\omega) P(\omega) e^{-i \omega D}}{1-|X(\omega)| e^{-i\left(\omega D-\Phi_{X}(\omega)\right)}}+\frac{E(\omega) Q(\omega) e^{-i \omega D}}{1-|Y(\omega)| e^{-i\left(\omega D-\Phi_{Y}(\omega)\right)}} \\
& \quad+\frac{E(\omega) R(\omega) e^{-i \omega D}}{1-|Z(\omega)| e^{-i\left(\omega D-\Phi_{Z}(\omega)\right)}} .
\end{aligned}
$$

We identify term by term the members of this equation. We take, for example,

$$
\frac{a_{1 k}}{\alpha_{1 k}+i\left(\omega-\omega_{1} k\right)} \simeq \frac{E(\omega) P(\omega) e^{-i \omega D}}{1-|X(\omega)| e^{-i\left(\omega D-\Phi_{X}(\omega)\right)}} .
$$

The resonance frequencies of each doublet $\omega_{1 k}, \omega_{2 k}$, and $\omega_{3 k}$ correspond to the minimum of the three denominators

$$
\begin{aligned}
& 1-|X(\omega)| e^{-i\left(\omega D-\Phi_{X}(\omega)\right)}, \\
& 1-|Y(\omega)| e^{-i\left(\omega D-\Phi_{Y}(\omega)\right)}, \\
& 1-|Z(\omega)| e^{-i\left(\omega D-\Phi_{Z}(\omega)\right)} .
\end{aligned}
$$

If we assume that moduli $|X(\omega)|,|Y(\omega)|$, and $|Z(\omega)|$ are close to one (this assumption is realistic because the propagation is weakly damped), we determine the values of $\omega_{1 k}$, 
$\omega_{2 k}$, and $\omega_{3 k}$ :

$$
\begin{aligned}
& \omega_{1 k}=\frac{\Phi_{X}\left(\omega_{1 k}\right)+2 k \pi}{D}, \\
& \omega_{2 k}=\frac{\Phi_{Y}\left(\omega_{2 k}\right)+2 k \pi}{D}, \\
& \omega_{3 k}=\frac{\Phi_{Z}\left(\omega_{3 k}\right)+2 k \pi}{D} .
\end{aligned}
$$

Taking $\omega=\omega_{1 k}+\epsilon$ with $\epsilon$ arbitrary small,

$$
\frac{a_{1 k}}{\alpha_{1 k}+i \epsilon} \simeq \frac{E\left(\omega_{1 k}+\epsilon\right) P\left(\omega_{1 k}+\epsilon\right) e^{-i \Phi_{X}\left(\omega_{1 k}+\epsilon\right)} e^{-i \epsilon D}}{1-\left|X\left(\omega_{1 k}+\epsilon\right)\right| e^{-i \epsilon D}} .
$$

A limited expansion of $e^{-i \epsilon D} \simeq 1-i \epsilon D+\theta\left(\epsilon^{2}\right)$ around $\epsilon=0$ (at the zeroth order for the numerator and at the first order for the denominator) gives

$$
\begin{gathered}
E\left(\omega_{1 k}+\epsilon\right) P\left(\omega_{1 k}+\epsilon\right) e^{-i \Phi_{X}\left(\omega_{1 k}+\epsilon\right)} e^{-i \epsilon D} \\
\simeq E\left(\omega_{1 k}\right) P\left(\omega_{1 k}\right) e^{-i \Phi_{X}\left(\omega_{1 k}\right)}, \\
1-\left|X\left(\omega_{1 k}+\epsilon\right)\right| e^{-i \epsilon D} \simeq 1-\left|X\left(\omega_{1 k}\right)\right|(1-i \epsilon D) .
\end{gathered}
$$

Assuming that $P(\omega)$ and $|X(\omega)|$ are locally constant (in the frequency domain), we identify term by term (the two members are considered as functions of the variable $\epsilon)$. We deduce the expressions of $|X(\omega)|,|Y(\omega)|$, and $|Z(\omega)|$ as a function of the amplitudes and decay times coefficients for each mode:

$$
\begin{gathered}
\left|X\left(\omega_{1 k}\right)\right|=\frac{1}{\alpha_{1 k} D+1}, \quad\left|Y\left(\omega_{2 k}\right)\right|=\frac{1}{\alpha_{2 k} D+1}, \\
\left|Z\left(\omega_{2 k}\right)\right|=\frac{1}{\alpha_{3 k} D+1} .
\end{gathered}
$$

We also get the relations

$$
\begin{aligned}
& E\left(\omega_{1 k}\right) P\left(\omega_{1 k}\right)=a_{1 k} D X\left(\omega_{1 k}\right), \\
& E\left(\omega_{2 k}\right) Q\left(\omega_{2 k}\right)=a_{2 k} D Y\left(\omega_{2 k}\right), \\
& E\left(\omega_{3 k}\right) Q\left(\omega_{3 k}\right)=a_{3 k} D Y\left(\omega_{3 k}\right) .
\end{aligned}
$$

From the measured signal, we estimate the modal parameters $a_{1 k}, a_{2 k}, a_{3 k}, \alpha_{1 k}, \alpha_{2 k}, \alpha_{3 k}, \omega_{1 k}, \omega_{2 k}$, and $\omega_{3 k}$. Using (A.17) and (A.20), we calculate $X, Y$, and $Z$. We still have 9 unknown variables $P, Q, R, E, C_{a}, C_{e}, F_{1}, F_{2}$, and $F_{3}$. But we also have a system of 9 equations ((A.6), (A.7), (A.8), (A.9), (A.10), (A.11), and (A.21)). Assuming that the two resonance frequencies are close and that the variables $P, Q, R, E, C_{a}$, $C_{e}, F_{1}, F_{2}, F_{3}, X, Y$, and $Z$ have a locally smooth behavior, we then express the waveguide parameters as function of the temporal parameters. For the sake of simplicity, we note $E_{k}=E\left(\omega_{1 k}\right)=E\left(\omega_{2 k}\right)$.

Using (A.6) and (A.9), we obtain $P_{k}+Q_{k}+R_{k}=X_{k}+$ $Y_{k}+Z_{k}$. Thanks to (A.21) we finally get the expression of the excitation signal at the resonance frequencies

$$
E_{k}=\frac{D\left(a_{1 k} X_{k}+a_{2 k} Y_{k}+a_{3 k} Z_{k}\right)}{X_{k}+Y_{k}+Z_{k}} .
$$

In the case of a two-coupled digital waveguide, the corresponding system admits analytical solutions (see [29]). But in the case of three-coupled digital waveguide, we have not found analytical expressions for variables $P, Q, R, C_{a}, C_{e}, F_{1}$, $F_{2}$, and $F_{3}$. We have then solved the system numerically.

\section{REFERENCES}

[1] J. Bensa, K. Jensen, R. Kronland-Martinet, and S. Ystad, "Perceptual and analytical analysis of the effect of the hammer impact on piano tones," in Proc. International Computer Music Conference, pp. 58-61, Berlin, Germany, August 2000.

[2] J. Bensa, F. Gibaudan, K. Jensen, and R. Kronland-Martinet, "Note and hammer velocity dependance of a piano string model based on coupled digital waveguides," in Proc. International Computer Music Conference, pp. 95-98, Havana, Cuba, September 2001.

[3] A. Askenfelt, Ed., Five Lectures on the Acoustics of the Piano, Royal Swedish Academy of Music, Stockholm, Sweden, 1990, Lectures by H. A. Conklin, Anders Askenfelt and E. Jansson, D. E. Hall, G. Weinreich, and K. Wogram, http://www.speech.kth.se/music/5_lectures/.

[4] S. Ystad, Sound modeling using a combination of physical and signal models, Ph.D. thesis, Université de la Méditérranée, Marseille, France, 1998.

[5] S. Ystad, "Sound modeling applied to flute sounds," Journal of the Audio Engineering Society, vol. 48, no. 9, pp. 810-825, 2000.

[6] A. Askenfelt and E. V. Jansson, "From touch to string vibrations. II: The motion of the key and hammer," Journal of the Acoustical Society of America, vol. 90, no. 5, pp. 2383-2393, 1991.

[7] A. Askenfelt and E. V. Jansson, "From touch to string vibrations. III: String motion and spectra," Journal of the Acoustical Society of America, vol. 93, no. 4, pp. 2181-2195, 1993.

[8] X. Boutillon, "Le piano: Modelisation physiques et developpements technologiques," in Congres Francais d'Acoustique Colloque C2, pp. 811-820, Lyon, France, 1990.

[9] P. Schaeffer, Traité des objets musicaux, Edition du Seuil, Paris, France, 1966.

[10] J. Laroche and J. L. Meillier, "Multichannel excitation/filter modeling of percussive sounds with application to the piano," IEEE Trans. Speech and Audio Processing, vol. 2, no. 2, pp. 329344, 1994.

[11] J. O. Smith III, "Physical modeling using digital waveguides," Computer Music Journal, vol. 16, no. 4, pp. 74-91, 1992.

[12] G. Borin, D. Rochesso, and F. Scalcon, "A physical piano model for music performance," in Proc. International Computer Music Conference, pp. 350-353, Computer Music Association, Thessaloniki, Greece, September 1997.

[13] B. Bank, "Physics-based sound synthesis of the piano," M.S. thesis, Budapest University of Technology and Economics, Budapest, Hungary, 2000, published as Report 54, Helsinki University of Technology, Laboratory of Acoustics and Audio Signal Processing, http://www.mit.bme.hu/ bank.

[14] J. O. Smith III, "Efficient synthesis of stringed musical instruments," in Proc. International Computer Music Conference, pp. 64-71, Computer Music Association, Tokyo, Japan, September 1993.

[15] J. O. Smith III and S. A. Van Duyne, "Commuted piano synthesis," in Proc. International Computer Music Conference, pp. 335-342, Computer Music Association, Banff, Canada, September 1995.

[16] S. A. Van Duyne and J. O. Smith III, "Developments for the commuted piano," in Proc. International Computer Music 
Conference, pp. 319-326, Computer Music Association, Banff, Canada, September 1995.

[17] A. Chaigne and A. Askenfelt, "Numerical simulations of struck strings. I. A physical model for a struck string using finite difference methods," Journal of the Acoustical Society of America, vol. 95, no. 2, pp. 1112-1118, 1994.

[18] X. Boutillon, "Model for piano hammers: Experimental determination and digital simulation," Journal of the Acoustical Society of America, vol. 83, no. 2, pp. 746-754, 1988.

[19] C. Valette and C. Cuesta, Mécanique de la corde vibrante, Traité des nouvelles technologies. Série Mécanique. Hermès, Paris, France, 1993.

[20] D. E. Hall and A. Askenfelt, "Piano string excitation V: Spectra for real hammers and strings," Journal of the Acoustical Society of America, vol. 83, no. 6, pp. 1627-1638, 1988.

[21] J. Bensa, S. Bilbao, R. Kronland-Martinet, and J. O. Smith III, "The simulation of piano string vibration: from physical model to finite difference schemes and digital waveguides," Journal of the Acoustical Society of America, vol. 114, no. 2, pp. 1095-1107, 2003.

[22] A. Chaigne and V. Doutaut, "Numerical simulations of xylophones. I. Time-domain modeling of the vibration bars," Journal of the Acoustical Society of America, vol. 101, no. 1, pp. 539-557, 1997.

[23] H. Fletcher, E. D. Blackham, and R. Stratton, "Quality of piano tones," Journal of the Acoustical Society of America, vol. 34, no. 6, pp. 749-761, 1962.

[24] D. A. Jaffe and J. O. Smith III, "Extensions of the KarplusStrong plucked-string algorithm," Computer Music Journal, vol. 7, no. 2, pp. 56-69, 1983.

[25] G. Weinreich, "Coupled piano strings," Journal of the Acoustical Society of America, vol. 62, no. 6, pp. 1474-1484, 1977.

[26] V. Välimäki, J. Huopaniemi, M. Karjalainen, and Z. Jánosy, "Physical modeling of plucked string instruments with application to real-time sound synthesis," Journal of the Audio Engineering Society, vol. 44, no. 5, pp. 331-353, 1996.

[27] B. Bank, "Accurate and efficient modeling of beating and twostage decay for string instrument synthesis," in Proc. Workshop on Current Research Directions in Computer Music, pp. 134137, Barcelona, Spain, November 2001.

[28] D. Rocchesso and J. O. Smith III, "Generalized digital waveguide networks," IEEE Trans. Speech and Audio Processing, vol. 11, no. 3, pp. 242-254, 2003.

[29] M. Aramaki, J. Bensa, L. Daudet, P. Guillemain, and R. Kronland-Martinet, "Resynthesis of coupled piano string vibrations based on physical modeling," Journal of New Music Research, vol. 30, no. 3, pp. 213-226, 2002.

[30] K. Steiglitz and L. E. McBride, "A technique for the identification of linear systems," IEEE Trans. Automatic Control, vol. 10, pp. 461-464, 1965.

[31] J. Beauchamp, "Synthesis by spectral amplitude and "brightness" matching of analyzed musical instrument tones," Journal of the Audio Engineering Society, vol. 30, no. 6, pp. 396406, 1982.

[32] S. McAdams, S. Winsberg, S. Donnadieu, G. de Soete, and J. Krimphoff, "Perceptual scaling of synthesized musical timbres: Common dimensions, specificities, and latent subject classes," Psychological Research, vol. 58, pp. 177-192, 1992.

[33] K. Jensen, "Musical instruments parametric evolution," in Proc. International Symposium on Musical Acoustics, pp. 319326, Computer Music Association, Mexico City, Mexico, December 2002.

[34] M. Laursen, C. Erkut, V. Välimäki, and M. Kuuskankara, "Methods for modeling realistic playing in acoustic guitar synthesis," Computer Music Journal, vol. 25, no. 3, pp. 38-49, 2001.
[35] K. Jensen, Timbre models of musical sounds, Ph.D. thesis, Department of Datalogy, University of Copenhagen, Copenhagen, Denmark, DIKU Tryk, Technical Report No 99/7, 1999.

Julien Bensa obtained in 1998 his Master's degree (DEA) in acoustics, signal processing, and informatics applied to music from the Pierre et Marie Curie University, Paris, France. He received in 2003 a Ph.D. in acoustics and signal processing from the University of Aix-Marseille II for his work on the analysis and synthesis of piano sounds using physical and signal models (available on line at http://www.lma.cnrs-mrs.fr/ bensa). He currently holds a postdoc position in the Laboratoire d'Acoustique Musicale, Paris, France, and works on the relation between the parameters of synthesis models of musical instruments and the perceived quality of the corresponding tones.

Kristoffer Jensen got his Master's degree in computer science at the Technical University of Lund, Sweden, and a DEA in signal processing at the ENSEEIHT, Toulouse, France. His Ph.D. was delivered and defended in 1999 at the Department of Datalogy, University of Copenhagen, Denmark, treating analysis/synthesis, signal processing, classification, and modeling of musical sounds. Kristoffer Jensen has a broad back-

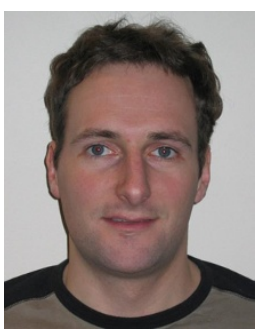
ground in signal processing, including musical, speech recognition and acoustic antenna topics. He has been involved in synthesizers for children, state-of the-art next-generation effect processors, and signal processing in music informatics. His current research topic is signal processing with musical applications, and related fields, including perception, psychoacoustics, physical models, and expression of music. He currently holds a position at the Department of Datalogy as Assistant Professor.

Richard Kronland-Martinet received a Ph.D. in acoustics from the University of Aix-Marseille II, France, in 1983. He received a "Doctorat d'Etat ès Sciences" in 1989 for his work on Analysis and synthesis of sounds using time-frequency and timescale representations. He is currently $\mathrm{Di}$ rector of Research at the National Center for Scientific Research (CNRS), Laboratoire de Mécanique et d'Acoustique in Marseille,

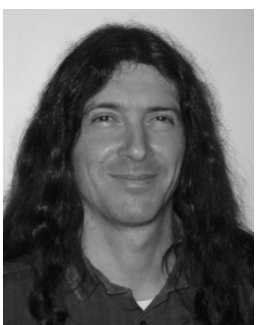
where he is the head of the group "Modeling, Synthesis and Control of Sound and Musical Signals." His primary research interests are in analysis and synthesis of sounds with a particular emphasis on musical sounds. He has recently been involved in a multidisciplinary research project associating sound synthesis processes and brain imaging techniques fonctional Nuclear Magnetic Resonance (fNRM) to better understand the way the brain is processing sounds and music. 